\title{
Desarrollo de los componentes sociales de la deportividad en futbolistas alevines
}

\section{Development of Sportsmanship Social Components in Juvenile Players}

\section{Desenvolvimento dos componentes sociais da desportivismo em jogadores fry}

\author{
J. Lamoneda Prietoํ, F.J. Huertas Delgado², L.G. Córdoba Caro y A.V. García Preciado
}

${ }^{1}$ Universidad de Extremadura, ${ }^{2}$ E.U. de Magisterio "La Inmaculada"

Resumen: El fútbol base demanda de un tratamiento ético que incluya no solo aspectos como el respeto, sino también: la cortesía, generosidad y ayuda. El objetivo del presente estudio fue elaborar un programa de intervención específicamente diseñado para jugadores de fútbol alevín centrado en la mejora de los aspectos sociales de la deportividad y analizar el cambio que genera. Participaron ciento veintiséis $(n=126)$ futbolistas federados de entre 10 y 12 ańos de edad. Se utilizó un diseño cuasi-experimental, con una fase de intervención de seis semanas de duración. Se administró la subescala factores sociales de la deportividad de la Escala Multidimensional de Orientaciones hacia la deportividad adaptada al fútbol base (Lamoneda, Huertas, Córdoba y García, 2014). En el análisis estadístico se recurrió a la prueba de Willcoxon y al test exacto de Fisher's. Se encontraron mejorías en aquellos jugadores que participaron en el programa en la cortesía hacia el oponente, atención e interés por el jugador lesionado y respeto al colegiado incluso cuando se equivoca. Se hallaron grandes limitaciones en la mejora de la capacidad del jugador para alentar al árbitro a que rectifique una situación injusta para el oponente. En el grupo control se apreció un descenso de la percepción de conductas de fair play. Los resultados obtenidos en este estudio permiten pensar que la inclusión de programas educativos en las planificaciones deportivas resulta beneficiosa como elemento preventivo frente a la tendencia hacia la pérdida de valores en el fútbol.

Palabras clave: programa de intervención, componentes sociales, deportividad, fútbol alevín.

Summary: Grassroots football demands ethical treatment that includes not only respect, but also courtesy, generosity and support. The objective of this study was to create an intervention program specifically tailored for young football players in order to improve the social aspects of the sportsmanship and analyse the changes that this might bring about. A hundred and twenty-six $(n=126)$ registered football players between the age of 10 and 12 participated in the research. A quasi-experimental design with a six-week-intervention phase was used. We tested the social factors in sportsmanship sub-scale of the Multi dimensional Sportspersonship in Juvenile Football Orientation Scale in Juvenile Football (Lamoneda, Huertas, Córdoba y García, 2014). A six- week long program was designed and developed. In statistical analysis, Wilcoxon test and Fisher's exact test were implemented. Improvements in players who participated in the program in regard to courtesy towards the opponent, care and concern for the injured player and respect towards the referee, even when he misruled, were noted. Major improvement limitations were found in regard to the player's ability to encourage the referee to rectify his unfair ruling towards the opponent. Sportsmanship evolution of players in the control group showed to be decreasing. Encouraging sportsmanship promotion findings in Grassroots football players are noted. Data shows that including educational programs in sport schedules is beneficial for moral development and values education; without which, educating through sport would be difficult.

Keywords: Intervention program, Social components, Sportsmanship, Juvenile football.

Resumo: $\mathrm{O}$ futebol de base exige um tratamento ético que inclua não apenas questốes como o respeito, mas também: cortesia, generosidade e apoio. O objetivo deste estudo foi desenvolver um programa de intervençấo especificamente desenhado para jogadores de futebol infantil com foco na melhoria dos aspetos sociais do desportivismo e analisar as mudanças que gera. Participaram cento e vinte seis $(n=126)$ jogadores federados entre 10 e 12 anos de idade. Foi utilizado un desenho quase-experimental com uma fase de intervenção de seis semanas de duraçáo. Administrou-se a sub-escala "fatores sociais do desportivismo" da "Escala Multidimensional de Orientaçôes" em direçấo ao desportivismo adaptada ao futebol de base (Lamoneda, Huertas, Córdoba e García, 2014). Na análise estatística foi utilizado o teste de Willcoxon e o teste exato de Fisher's. Encontraram-se melhorias, naqueles jogadores que participaram no programa, na cortesía para com o jogador lesionado e respeito pelo árbitro, mesmo quando estava errado. Foram encontradas grandes limitaçóes na melhoria da capacidade do jogador para incentivar o árbitro para corrigir uma situaçâo injusta para o adversário. No grupo central foi apreciada uma redução da perceção de comportamentos de fair play. Os resultados obtidos neste estudo levam-nos a crer que a inclusão de programas educativos nas programaçóes desportivas é benéfico como um elemento de prevenção contra a tendência em direção à perda de valores no futebol.

Palavras-chave: Programa de intervenção, componentes sociais, desportivismo, futebol infantil.

\section{Introducción}

El juego limpio y la deportividad sintetizan el código ético en el deporte. Integran valores relacionados con la ética in-

\footnotetext{
Dirección para correspondencia [Correspondence address]: Javier Lamoneda Prieto. Funcionario y profesor del IES Seritium. Avda. Ámsterdam s/n. 11405. Jerez de la Frontera, Cádiz (España). E-mail: educacionfisicajlp@gmail.com
}

dividual y social (Aristóteles, trad. 1972). Los valores que integra la ética social se componen: en primer lugar, del respeto a las normas del juego, adversarios y al árbitro (Comité Olímpico Internacional, 2001); en segundo lugar, principios fundamentales de justicia (Comité Internacional de Fair Play, 2012): ser honesto en todo momento del juego y garantizar el 
cumplimiento de la justicia para todos, sea o no beneficioso para los intereses personales; y en tercer lugar, una orientación a maximizar la experiencia agradable de todos los participantes (Lemyre, Roberts y Ommudsen, 2002): felicitar y reconocer el buen juego del adversario, interesarse por el jugador lesionado, tener una actitud digna tanto en la victoria como en la derrota, prestar material deportivo, ser compasivo con el jugador que llega tarde (Vallerand, Brière, Blanchard y Provencher, 1997) incluso, alegrarse con la victoria del otro por valorar su esfuerzo y competitividad durante el encuentro (Hechenberger, 2004).

A pesar del legado de buenas intenciones que incluye el término deportividad, la teoría dista bastante de la realidad y los comportamientos antisociales cotejan con alta frecuencia al fútbol (Kavussanu, Stamp, Slade y Ring, 2009). Para verificar esta situación, previo a este trabajo se realizó un análisis exploratorio de conductas de fair play con una muestra de treinta equipos de similar ubicación geográfica. Los resultados revelaban entre otros datos: carencias en relación con la cortesía hacia el oponente durante y al finalizar los encuentros (Lamoneda, García, Córdoba y Vizuete, 2014).

Para solventar los problemas éticos a los que se expone el deporte base han proliferado con los años numerosos programas formativos. Los marcos teóricos que han sustentado los programas de intervención basados en el desarrollo de la Responsabilidad en el ámbito deportivo han sido: la Teoría de la autodeterminación, el Modelo jerárquico de Motivación intrínseca y extrínseca, el Modelo trans-contextual de la motivación y la Teoría de las metas sociales (Belendo, Ferriz-Morell y Moreno-Murcia, 2012). Se caracterizan por ser fundamentalmente de tipo preventivo e incluir actividades de reflexión, debate, disuasión, concienciación y difusión dirigidos a los grupos sociales implicados (Sáenz, Gimeno, Gutiérrez y Garay, 2012).

En este estudio se recurrió a la utilización de la sub-escala "factores sociales de la deportividad" de la "Escala Multidimensional de Orientaciones hacia la Deportividad adaptada al fútbol alevín (Lamoneda, Huertas, Córdoba y García, 2014) al tratarse de una herramienta validada para su utilización en el fútbol alevín, con la que se iba a realizar el estudio; y por ajustarse al plan de trabajo que se iba a desarrollar, ya que estructuraba bien el concepto de deportividad y permitía valorar actitudes prodeportivas.

El objetivo del presente estudio fue elaborar un programa de intervención específicamente diseńado para jugadores de fútbol alevín centrado en la mejora de los aspectos sociales de la deportividad y analizar el cambio que genera. Por tanto, se formuló como hipótesis que tras la aplicación del Programa de Educación en Valores los jugadores que participaron (grupo experimental) mejorarían la componente social de la deportividad respecto a los que no siguieron ningún tratamiento moral (grupo control).

\section{Método}

\section{Participantes}

126 futbolistas con una edad comprendida entre diez y doce años $(M=10.99, S D=0,67)$. En la fase de intervención diferenciamos dos grupos: experimental, compuesto por sesenta y un jugadores (con el que se desarrolla el programa) y control, de sesenta y cinco participantes (que no recibe ningún tratamiento).

Se realizó un estudio con los clubes y asociaciones deportivas de categoría alevín y con sede en una población de 200.000 habitantes al sur de España. De los veintiún clubes que cumplían estos requisitos se seleccionaron doce.

Proceso de selección de la muestra. Se fraguó en dos períodos: temporada 2011-2012 y 2012-2013. En el primer período se recurrió a la técnica de muestreo sistémico y por cúmulos y en el segundo al muestreo por conveniencia con el fin de mejorar la representatividad de la muestra inicial que no incluía equipos pertenecientes a clubes ubicados en barrios en "riesgo de exclusión social" ni "filiales de clubes inscritos en la Liga de Fútbol Profesional".

En la fase de intervención diferenciamos dos grupos: experimental, compuesto por seis equipos ya formados $(n=61)$, a los que se le administró el programa, y control, constituido por seis clubes $(n=65)$ también constituidos previo al estudio, que no recibió tratamiento.

Sesgo de selección. Con el fin de valorar la homogeneidad de las muestras se tuvieron en cuenta variables socio-demográficas (sexo, edad, condición socioeconómica de la familia de origen y rendimiento del equipo a lo largo de la temporada). Previo al estudio se analizó la posible influencia del rendimiento y condición socio-económica en el programa. Los resultados revelaron que no existían diferencias significativas entre futbolistas de diferente condición socio-económica ni entre deportistas de distinto rendimiento y que por consiguiente no eran factores limitantes para una posible intervención con la muestra analizada (Lamoneda, 2014). No se realizó un estudio diferenciado de sexo debido al reducido número de chicas que incluía la muestra (1,58\%). 
Tabla 1. Características socio-demográficas de la muestra de estudio.

\begin{tabular}{|c|c|c|c|c|c|c|c|}
\hline Muestra & & $\begin{array}{c}\text { Total } \\
\mathrm{n}=126\end{array}$ & $\%$ & $\begin{array}{c}\text { Experimental } \\
\mathrm{n}=61\end{array}$ & $\%$ & $\begin{array}{c}\text { Control } \\
\mathrm{n}=65\end{array}$ & $\%$ \\
\hline \multirow{3}{*}{$\begin{array}{l}\text { Edad } \\
\text { (años) }\end{array}$} & 10 & 29 & 23,02 & 10 & 16,39 & 19 & 29,23 \\
\hline & 11 & 70 & 55,56 & 31 & 50,82 & 39 & 60 \\
\hline & 12 & 27 & 21,43 & 20 & 32,79 & 7 & 10,77 \\
\hline \multirow{3}{*}{$\begin{array}{l}\text { Sexo } \\
(h / m)\end{array}$} & $\mathrm{H}$ & 124 & 98,72 & 59 & 96,72 & 65 & 100 \\
\hline & M & 2 & 1,58 & 2 & 3,27 & 0 & 0 \\
\hline & Parados & 10 & 7,93 & 4 & 6,55 & 6 & 9,23 \\
\hline \multirow[t]{2}{*}{ Condición socio-económica } & Obreros & 60 & 47,61 & 29 & 47,54 & 31 & 47,69 \\
\hline & Pequeños Empresarios & 27 & 21,42 & 10 & 16,39 & 17 & 26,15 \\
\hline \multirow[t]{3}{*}{ (padre Imadre) } & Técnicos & 14 & 11,11 & 8 & 13,11 & 6 & 9,23 \\
\hline & Profesionales & 15 & 11,90 & 10 & 16,39 & 5 & 7,69 \\
\hline & Muy bajo & 29 & 25 & 29 & 50 & 0 & 0 \\
\hline \multirow{4}{*}{ Rendimiento } & Bajo & 11 & 8,33 & 0 & 0 & 11 & 16,66 \\
\hline & Medio & 14 & 8,33 & 0 & 0 & 14 & 16,66 \\
\hline & Alto & 32 & 25 & 12 & 16,66 & 20 & 33,33 \\
\hline & Muy alto & 40 & 33 & 20 & 33 & 20 & 33 \\
\hline
\end{tabular}

Nota: Condición socio-económica: se registró entre los dos progenitores la profesión de mayor rango en la escala. Rendimiento: se registró el rendimiento del equipo al que pertenecía el jugador en una escala de muy bajo, “último, penúltimo o antepenúltimo", bajo, "4º , 50 o 6º en orden inverso a la clasificación general”, medio, "todos los que no se incluyen en el resto de categorías", alto "40, 5० o 60" y muy alto, "10, $2^{\circ}$ o $3^{\circ}$ ".

Tabla 2. Prueba U de Mann-Whitney de muestras independientes.

\begin{tabular}{lcc}
\hline & Sig. & $\begin{array}{c}\text { Decisión en } \\
\text { relación con la } \\
\text { Hipótesis nula }\end{array}$ \\
\hline 1. Dar la mano al vencedor &, 120 & Retener \\
2. Dar la mano al entrenador oponente &, 689 & Retener \\
3. Felicitar al oponente &, 135 & Retener \\
4. Animar al perdedor &, 104 & Retener \\
5. Obedecer al árbitro &, 244 & Retener \\
6. Respetar al árbitro &, 512 & Retener \\
7. Permitir jugar al que llega tarde &, 855 & Retener \\
8. Preocuparse por el lesionado &, 379 & Retener \\
9. Rectificar una situación injusta &, 915 & Retener \\
10. Prestar material deportivo &, 102 & Retener \\
11. Rechazar un gol y atender al lesionado &, 335 & Retener \\
Factores sociales de la deportividad &, 460 & Retener \\
\hline
\end{tabular}

Se valoró estadísticamente la homogeneidad de la muestra con los datos obtenidos en pretest a través la prueba $U$ de Mann-Whitney de muestras independientes. Partiendo de la hipótesis nula de contraste: "la distribución es la misma entre las categorías de experimental o control" caben dos decisiones en la interpretación de los resultados aportados por la prueba: primero, rechazar la hipótesis nula, si existen dife- rencias significativas $(\leq, 05)$ y considerar que los grupos no son homogéneos o segundo, retener la hipótesis nula $(\geq, 05)$ y afirmar que las muestras son homogéneas. Como puede observarse en la tabla 2 en el $100 \%$ de los ítems que componen la sub-escala "factores sociales de la deportividad" los valores obtenidos nos permiten afirmar que no existían inicialmente diferencias entre el grupo control y experimental.

\section{Instrumentos}

Para la recogida de datos se recurrió a la utilización de la subescala 'componentes sociales de la deportividad' de la versión española adaptada al fútbol alevín de la Multidimensional Sportspersonship Orientations Scale (MSOS) de Vallerand et al. (1997) validada por Lamoneda et al. (2014b). La sub-escala se compone de 11 ítems relacionados con: las convecciones sociales, el respeto al árbitro y a los oponentes. Las respuestas se recogen en una escala tipo Likert con un rango de 1 a 5 , siendo 1, "no se corresponde conmigo en absoluto"; 3, "se corresponde conmigo en parte" y 5 , "se corresponde exactamente conmigo".

En el estudio de Lamoneda et al. (2014b) los valores del coeficiente de fiabilidad fueron ,701 en pre-test y ,722 en post-test para la sub-escala 'componentes sociales de la deportividad', mostrando una consistencia interna satisfactoria. 


\section{Procedimiento}

El diseño de este trabajo se ubica dentro del paradigma experimental, en concreto siguiendo una metodología cuasi experimental de grupo control no equivalente con medidas pretest y postest en contextos reales. Se trata de un modelo muy utilizado en la investigación social (Ato y Vallejo, 2007; Cook y Campbel, 1979; Ramos, 2011).

El estudio se llevó a cabo entre abril de 2011 y abril de 2013 en dos etapas: fase previa (fundamentación teórica, diseño del programa y del manual) e intervención, que se fraguó en dos temporadas: 2012 y 2013. La estructura del programa de intervención en ambos períodos se componía de: un período preparatorio y otro de intervención (figura 1).

Periodo preparatorio. En el período previo a la intervención se presentó el proyecto a las entidades deportivas y se solicitaron autorizaciones a los representantes legales de los participantes. En el programa se contó con la colaboración de doce Técnicos de Ciclo Formativo de Grado Superior en Animación y Actividad Física, que recibieron una formación previa de tres semanas de duración. Se establecieron grupos de tres monitores por equipo. Previo a la intervención educativa el coordinador del programa estableció una reunión individual con el entrenador del equipo y una segunda con entrenador y monitores.

Evaluación inicial. A los doce equipos que conformaban la muestra se les pasó la Escala Multidimensional de Orientaciones hacia la Deportividad adaptada al fútbol alevín (MSOSF). La evaluación se llevó a cabo con ocho clubes entre noviembre y diciembre de 2011 y con los cuatro clubes restantes entre noviembre y diciembre de 2012. La administración de la MSOS-F se realizó bajo la presencia del investigador principal y siempre que hubo la posibilidad, en el vestuario del equipo. Los casos en los que no se contó con esta ubicación se habilitaron otras zonas como: bancos o vallas situadas en zonas colindantes al campo de entrenamiento. Durante la administración de la escala se les comunicó verbalmente a los jugadores que debían dar su opinión personal sobre cada una de las situaciones planteadas con sinceridad y sin dejar ninguna cuestión sin responder. A cada jugador se le facilitó el material necesario para cumplimentar la escala. Los cuestionarios fueron aplicados en grupos de entre siete y catorce participantes $(M=10,54 ; S D=2,16)$. Durante su administración se les leyó cada ítem en voz alta y se les dejó tiempo para que respondiesen a cada cuestión. La principal duda que le generaba la herramienta fue graduar la respuesta en una escala (de 1 a 5) en lugar de en dos (si o no). Se insistió que lo correcto era contestar de acuerdo con lo que ellos pensaban y que no siempre era necesario utilizar las respuestas extremas (1 ó 5).

Fase de intervención. Se iniciaba con una sesión de presentación con los jugadores en la que estaban presentes los monitores y el entrenador principal. El programa de juego limpio se prolongó a seis semanas con cada entidad deportiva. Se acordó con los en- trenadores: dedicar veinte minutos semanales al trabajo de educación en valores, en la que se orientaba al deportista a adquirir un compromiso; realizar un seguimiento del mismo en la segunda sesión de entrenamiento y durante la competición; y valorar los logros una semana posterior a la adquisición de los mismos. Siempre que fue posible la intervención se realizó: el primer día de la semana antes de empezar en entrenamiento.

Evaluación final. La MSOS-F se pasó durante el meses de abril de 2012 y 2013 con el fin de comparar los resultados finales con los iniciares y poder así valorar la eficacia de la intervención.

Secuenciación de objetivos de enseñanza. Para establecer una secuencia coherente en la transmisión de valores a lo largo de la temporada se tomó como referencia los trabajos llevados a cabo desde 1970 por Donald Hellison en Canadá: Programa de responsabilidad personal y social (Hellison, 1995). Los cinco niveles de Hellison $-1^{\circ}$ respeto, $2^{\circ}$ participación, $3^{\circ}$ autogestión, $4^{\circ}$ ayuda y $5^{\circ}$ aplicación fuera del club- fueron adaptados al fútbol alevín por: $1^{\circ}$ confianza, $2^{\circ}$ respeto, $3^{\circ}$ cortesía y $4^{\circ}$ ayuda.

Estrategia metodológica. Este programa se basa tanto en las teorías de aprendizaje social como del desarrollo estructural. Dentro de las teorías de aprendizaje social: en la utilización de modelos deportivos, que se trata de un método de contrastada eficacia en el desarrollo moral (Meier y Koenen, 2010) y en el desarrollo de competencias autorreguladoras (pedagogía de compromisos). Esta última estratégica tiene como meta lograr el auto control de la conducta. Pretende que el deportista sea dueño de sus propios actos, que llegue a existir coherencia entre sus pensamientos y sus actos. Según Kohlberg la "norma colectiva" es un medio adecuado para la mejora de la "atmósfera moral", aspecto de crítica influencia en el comportamiento moral de las personas. Esta línea metodológica ha sido utilizada en otros programas cómo: "Ir en busca de la meta" (Danish, 1997), o "Delfos" (Cecchini et al., 2009) que se centraron en la propuesta de metas y los procesos de transferencia son pilares básicos. Además, se tomó como referencia el programa "Sport4Pace" (Hechenberger, 2001) que fue aplicado en la fase preparatoria a la Eurocopa de Fútbol de 2008.

Diferenciamos cuatro fases (modificado de: Ruiz, Rodríguez, Martinek, Schilling, Durán, y Jiménez, 2006; y Hechenberger, 2001): (1) reunión, (2) reflexión y adquisición de compromisos, (3) acción y (4) reflexión. Esta secuencia tenía una duración semanal en la que las dos primeras fases se trabajaban en una sesión, el día estipulado por los clubes fue: lunes o martes. La tercera fase se desarrollaba en un período entre dos reuniones. Para la mayoría de los clubes incluía la segunda sesión de entrenamiento del equipo (miércoles o jueves) y la competición de liga (sábado o domingo). Tan solo dos clubes entrenaban tres días en semana. Finalmente, la cuarta fase se ubicaba al comienzo de la segunda sesión, exactamente una semana después de la primera reunión. 
Figura 1. Procedimiento.

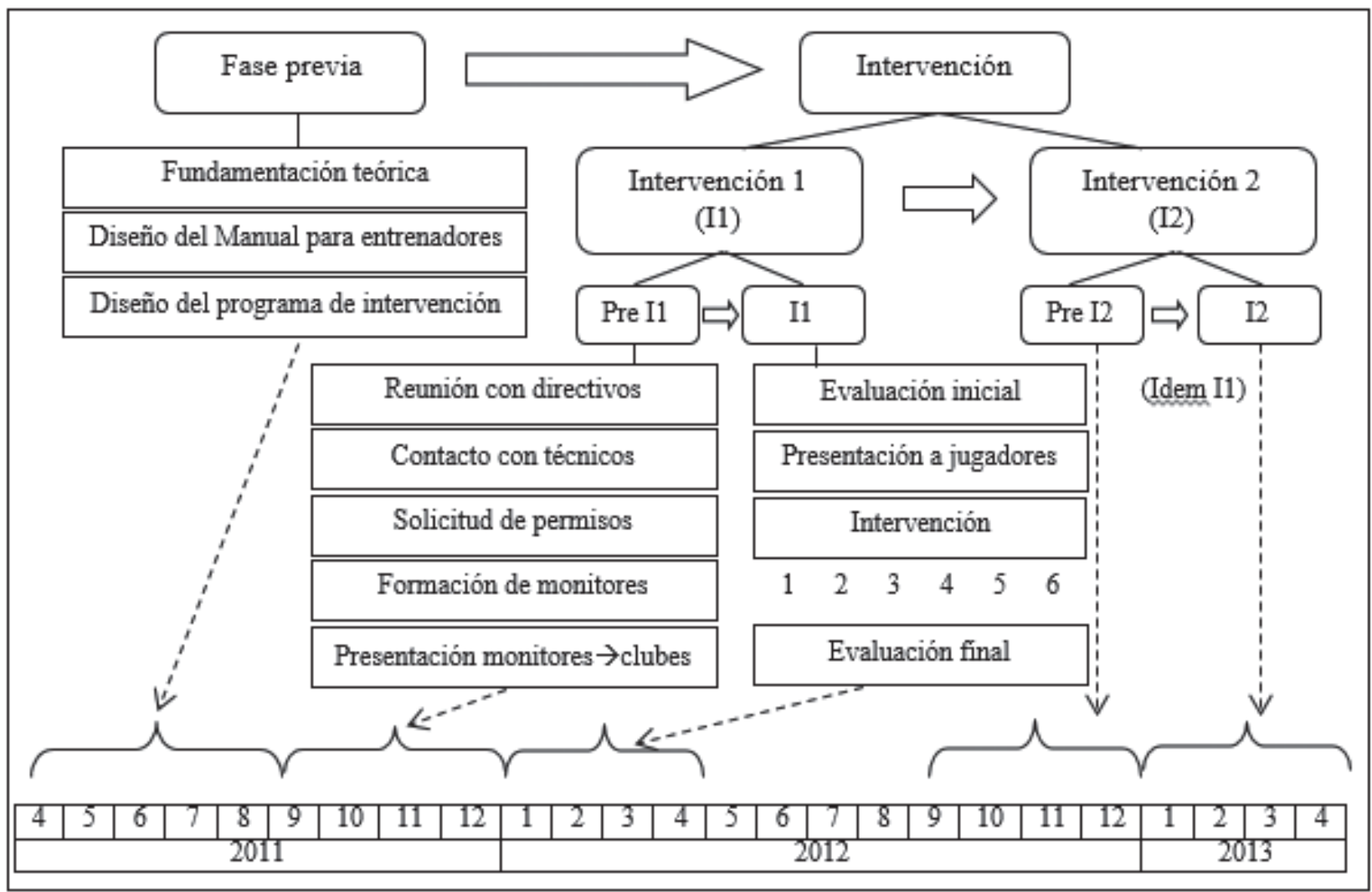

(1) Reunión en el vestuario. Se trataba que los jugadores realizasen un análisis de las propias conductas y las comparasen con las formas de comportamiento deportivo valioso en relación con cada uno de los temas del programa: "juega limpio", "respeta al árbitro”, "discúlpate", etc. En esta fase se recurrió a las siguientes técnicas de enseńanza: a) Directivas, entre las que destaca la inculcación de valores, propios de las teorías de aprendizaje social (Bandura, 1986). Se llegaba a la reflexión a partir de un discurso fundamentado por parte del técnico deportivo en el que incluía modelos de buen deportista. b) Indagativas, enfocadas en el desarrollo del juicio moral, siguiendo a la teoría del desarrollo estructural de Kohlberg (1969). Las técnicas más utilizadas en este caso fueron el comentario crítico de textos (comprensión crítica) y los diálogos clarificadores: hojas de valores, frases inacabadas y preguntas esclarecedoras (clarificación de valores).

(2) Reflexión personal y adquisición de compromisos. A cada uno de los participantes se les entregó al iniciar el programa una ficha de registro en la que debían anotar los compromisos semanales que adquirían y las experiencias positivas que iban realizando durante la temporada. Al finalizar cada sesión los compromisos se guardaban en una caja junto con los recursos materiales facilitados por el programa (material de oficina y dossier del programa para el entrenador con las sesiones desarrolladas).

(3) Acción. Puesta en práctica los compromisos adquiridos en los entrenamientos y competición.

(4) Reflexión. La revisión de compromisos y refuerzo de conductas se realizaba semanalmente al empezar la reunión del equipo. Además, se le animaba al entrenador a que incluyese tiempos de reflexión al finalizar cada entrenamiento y partido.

\section{Análisis estadístico}

Se utilizó el programa estadístico SPSS 20.0 para Windows. En el estudio diferenciamos dos análisis: intra e inter grupo. En el primero se valoró como el grupo control y el experimental de forma independiente evolucionaban en la temporada, para ello se empleó la prueba de Willcoxon. Con el segundo la pretensión era realizar un estudio comparativo entre el control y experimental, para lo cual se recurrió al test exacto de Fisher's. Se analizaron tres aspectos: primero, los jugadores que mejoraron tras la intervención: incrementar los puntajes; segundo, los que tuvieron una evolución positiva: pasar de una postura 
no favorable (1-3 puntos) a positiva ( $\geq 4$ puntos); y tercero, los que involucionaron: de valores positivos a negativos o neutros.

\section{Resultados}

\section{Análisis intra-grupo}

Grupo experimental. En la tabla 3 se observa cómo los valores promedio de los participantes del grupo experimental mejoraron significativamente $(p \leq, 05)$.

Al concluir el estudio los jugadores del grupo experimental obtuvieron cinco ítems con valores positivos $(M \geq 4)$ : 1 (dar la mano al vencedor), 4 (animar al perdedor), 5 (obedecer al árbitro), 7 (permitir jugar al que llega tarde) y 8 (preocuparse por el lesionado) (tabla 3).

Las puntuaciones contrarias a la deportividad $(M<4)$ en postest fueron: 2 (dar la mano al entrenador oponente al finalizar el partido), 3 (felicitar al otro equipo), 6 (respetar al árbitro cuando se equivoca), 9 (rectificar una situación injusta para el otro equipo), 10 (prestar material deportivo al otro equipo) y 11 (rechazar un gol y atender al lesionado).

Al estudiar los resultados en los que se detectaron diferen- cias significativas entre valores iniciares y finales apreciamos cómo el experimental incrementó sus puntuaciones en cuatro ítems: 2 (dar la mano al entrenador oponente), 3 (felicitar al oponente), 4 (animar al perdedor) y 11 (rechazar un gol para atender al lesionado) y tan solo empeoró en uno: 9 (rectificar una situación injusta) (tabla 3).

Grupo control. Los jugadores del grupo control empeoraron significativamente $(p \leq, 05)$ los valores promedio. Obtuvieron al consumar la temporada diez ítems con puntuaciones opuestas al juego limpio $(M<4)$, seis de los cuales con valores claramente contrarios a la deportividad $(M<3): 2$ (dar la mano al entrenador oponente), 3 (felicitar al oponente), 4 (animar al perdedor), 6 (respetar al árbitro cuando se equivoca), 9 (rectificar una situación injusta para el oponente) y 10 (prestar material deportivo). Tan solo se hallaron orientaciones prodeportivas en un ítem: 1 (dar la mano al vencedor) (tabla 3).

Se apreciaron cinco casos en los que los valores del grupo control decrecieron significativamente $(p \leq, 05)$ : 4 (animar al perdedor), 6 (respetar al árbitro cuando se equivoca), 7 (permitir jugar al que llega tarde), 8 (preocuparse por el lesionado) y 9 (rectificar una situación injusta para el oponente). No se encontraron casos en los que el grupo control incrementase sus valores.

Tabla. 3. Análisis intra-grupo. Estadísticos descriptivos y prueba de Willcoxon.

\begin{tabular}{|c|c|c|c|c|c|c|c|c|}
\hline \multirow{2}{*}{ Descriptivos } & \multicolumn{4}{|c|}{ Experimental } & \multicolumn{4}{|c|}{ Control } \\
\hline & Pre test & Post test & P Valor & Incremento & Pre test & Post test & P Valor & Incremento \\
\hline Dar la mano al vencedor & $\begin{array}{c}4,00 \\
\pm 1,17\end{array}$ & $\begin{array}{c}4,16 \\
\pm 1,16\end{array}$ & ,267 & 0,16 & $\begin{array}{c}4,26 \\
\pm 1,20\end{array}$ & $\begin{array}{c}4,03 \\
\pm 1,39\end{array}$ & ,127 & $-0,23$ \\
\hline Dar la mano al entrenador oponente & $\begin{array}{r}2,36 \\
\pm 1,63\end{array}$ & $\begin{array}{r}3,44 \\
\pm 1,49\end{array}$ &, $000^{*}$ & 1,08 & $\begin{array}{c}2,35 \\
\pm 1,40\end{array}$ & $\begin{array}{c}2,62 \\
\pm 1,43\end{array}$ &, 067 & 0,26 \\
\hline Felicitar al oponente & $\begin{array}{c}2,49 \\
\pm 1,60\end{array}$ & $\begin{array}{c}3,31 \\
\pm 1,15\end{array}$ &, $001^{*}$ & 0,82 & $\begin{array}{r}2,86 \\
\pm 1,55\end{array}$ & $\begin{array}{c}2,62 \\
\pm 1,23\end{array}$ &, 157 & $-0,25$ \\
\hline Animar al perdedor & $\begin{array}{c}3,79 \\
\pm 1,47\end{array}$ & $\begin{array}{c}4,33 \\
\pm 1,03\end{array}$ &, $026^{*}$ & 0,54 & $\begin{array}{r}4,05 \\
\pm 1,53\end{array}$ & $\begin{array}{r}2,63 \\
\pm 1,49\end{array}$ &, $000^{*}$ & $-1,41$ \\
\hline Obedecer al árbitro & $\begin{array}{c}4,26 \\
\pm 0,91\end{array}$ & $\begin{array}{c}4,10 \\
\pm 1,09\end{array}$ & ,333 & $-0,16$ & $\begin{array}{c}3,91 \\
\pm 1,31\end{array}$ & $\begin{array}{c}3,63 \\
\pm 1,35\end{array}$ & ,118 & $-0,28$ \\
\hline Respetar al árbitro cuando se equivoca & $\begin{array}{c}3,03 \\
\pm 1,28\end{array}$ & $\begin{array}{c}3,38 \\
\pm 1,19\end{array}$ &, 147 & 0,35 & $\begin{array}{c}3,18 \\
\pm 1,25\end{array}$ & $\begin{array}{r}2,55 \\
\pm 1,32\end{array}$ &, $002^{*}$ & $-0,63$ \\
\hline Permitir jugar al que llega tarde & $\begin{array}{c}4,10 \\
\pm 1,03\end{array}$ & $\begin{array}{c}4,23 \\
\pm 1,06\end{array}$ & ,385 & 0,13 & $\begin{array}{r}3,89 \\
\pm 1,55\end{array}$ & $\begin{array}{c}3,32 \\
\pm 1,54\end{array}$ &, $028^{*}$ & $-0,57$ \\
\hline Preocuparse por el lesionado & $\begin{array}{c}4,03 \\
\pm 1,00\end{array}$ & $\begin{array}{c}4,10 \\
\pm 1,27\end{array}$ & ,292 & 0,07 & $\begin{array}{r}4,09 \\
\pm 1,17\end{array}$ & $\begin{array}{c}3,29 \\
\pm 1,16\end{array}$ &, $001^{*}$ & $-0,80$ \\
\hline Rectificar una situación injusta & $\begin{array}{c}1,85 \\
\pm 1,29\end{array}$ & $\begin{array}{c}1,44 \\
\pm 1,06\end{array}$ &, $015^{*}$ & $-0,41$ & $\begin{array}{c}1,75 \\
\pm 1,17\end{array}$ & $\begin{array}{c}1,35 \\
\pm 0,87\end{array}$ &, $018^{*}$ & $-0,40$ \\
\hline Prestar material deportivo & $\begin{array}{c}2,93 \\
\pm 1,67\end{array}$ & $\begin{array}{c}3,25 \\
\pm 1,61\end{array}$ &, 175 & 0,31 & $\begin{array}{r}2,45 \\
\pm 1,46\end{array}$ & $\begin{array}{c}2,20 \\
\pm 1,37\end{array}$ & ,236 & $-0,25$ \\
\hline Rechazar un gol y atender al lesionado & $\begin{array}{c}3,28 \\
\pm 1,42\end{array}$ & $\begin{array}{c}3,79 \\
\pm 1,37\end{array}$ &, $013^{*}$ & 0,51 & $\begin{array}{r}3,49 \\
\pm 1,47\end{array}$ & $\begin{array}{c}3,08 \\
\pm 1,48\end{array}$ &, 087 & $-0,41$ \\
\hline Factores sociales de la deportividad & $\begin{array}{c}3,16 \\
\pm 1,02\end{array}$ & $\begin{array}{l}3,82 \\
\pm 0,76\end{array}$ &, $000^{*}$ & 0,66 & $\begin{array}{c}3,39 \\
\pm 0,89\end{array}$ & $\begin{array}{c}2,97 \\
\pm 0,81\end{array}$ &, $000^{*}$ & $-0,42$ \\
\hline
\end{tabular}

Nota: Los datos que se presentan en una escala liket donde 5 representa un valor muy positivo de juego limpio y 1 negativo. Se utiliza el color "gris" para expresar datos en los que mejora significativamente $(p \leq, 05)$ el experimental al control y en "negro" el control al experimental. 


\section{Análisis inter-grupo}

Como puede apreciarse en la tabla 4, los resultados obtenidos en el estudio de valores promedio revelan cómo el grupo experimental mejoró y evolucionó significativamente más que el control $(p \leq, 05)$. No se obtuvieron diferencias significativas en el análisis de valores regresivos.

El análisis pormenorizado de cada ítem revela cómo tanto en el ítem no 3 (felicitar al oponente), el no 4 (animar al perdedor), como en el no 6 (respetar al árbitro cuando se equivoca) se dio la casuística de que el grupo experimental mejoró más que el control $(p \leq, 05)$ y a su vez el número de jugadores del experimental que partían de una postura contraria a la deportividad (valores 1-3) evolucionó mejor que los del control hacia orientaciones prodeportivas (valores 4-5).

Se obtuvieron dos casos en los que el grupo experimental mejoró más que el control y a su vez el control empeoró sig- nificativamente más que el experimental $(p \leq, 05)$ : 8 (preocuparse por el jugador lesionado) y 11 (rechazar un gol y atender al lesionado) (tabla 4).

Se encontraron dos ítems en los que la evolución fue significativamente positiva en el experimental respecto al control: 1 (dar la mano al vencedor) y 2 (dar la mano al entrenador oponente) (tabla 4).

Los jugadores del grupo control empeoraron más que el experimental en los ítems: 6 (respetar al árbitro cuando se equivoca), 7 (permitir jugar al que llega tarde), 8 (preocuparse por el lesionado) y 9 (rectificar una situación injusta para el oponente).

No se observaron diferencias entre el experimental y control en los ítems: 5 (obedecer al árbitro), 9 (rectificar una situación injusta para el oponente) y 10 (prestar material deportivo).

Tabla 4. Análisis inter-grupo. Test exacto de Fisher's.

\begin{tabular}{lccccccccc}
\hline & \multicolumn{3}{c}{ Mejora } & \multicolumn{3}{c}{ Evolución positiva } & \multicolumn{3}{c}{ Evolución negativa } \\
\cline { 2 - 9 } & Exp. & Cont. & Sig. & Exp. & Cont. & Sig. & Exp. & Cont. & Sig. \\
\hline 1. Dar la mano al vencedor & 32,79 & 27,69 &, 539 & 24,59 & 6,15 &, $005^{*}$ & 6,56 & 10,77 &, 532 \\
2. Dar la mano al entrenador oponente & 52,46 & 38,46 &, 064 & 36,07 & 15,38 &, $000^{*}$ & 4,92 & 6,15 & 1 \\
3. Felicitar al oponente & 55,74 & 33,85 &, $003^{*}$ & 24,59 & 4,62 &, $002^{*}$ & 11,48 & 18,46 &, 325 \\
4. Animar al perdedor & 44,26 & 9,23 &, $000^{*}$ & 27,87 & 4,62 &, $000^{*}$ & 9,84 & 43,08 &, $000^{*}$ \\
5. Obedecer al árbitro & 16,39 & 20 &, 581 & 11,48 & 12,31 & 1 & 16,39 & 20 &, 650 \\
6. Respetar al árbitro cuando se equivoca & 40,98 & 15,38 &, $000^{*}$ & 21,31 & 6,15 &, $000^{*}$ & 19,67 & 26,15 &, 406 \\
7. Permitir jugar al que llega tarde & 32,79 & 21,54 &, 113 & 18,03 & 9,23 &, 192 & 13,11 & 38,46 &, $002^{*}$ \\
8. Preocuparse por el lesionado & 42,62 & 21,54 &, $002^{*}$ & 14,75 & 9,23 &, 414 & 13,11 & 41,54 &, $001^{*}$ \\
9. Rectificar una situación injusta & 9,84 & 10,77 & 1 & 3,23 & 1,54 &, 610 & 11,48 & 7,69 &, 552 \\
10. Prestar material deportivo & 37,70 & 27,69 &, 176 & 18,03 & 9,23 &, 194 & 11,48 & 16,92 &, 451 \\
11. Rechazar un gol y atender al lesionado & 42,62 & 27,69 &, $038^{*}$ & 26,23 & 13,85 &, 117 & 11,48 & 27,69 &, $026^{*}$ \\
\hline Factores sociales de la deportividad & 37,11 & 23,07 &, $032^{*}$ & 20,57 & 8,39 &, $041^{*}$ & 11,77 & 23,36 &, 103 \\
\hline Nota: Valores expresados en porcentajes. Se utiliza el color "gris" para expresar datos en los que mejora significativamente $(p \leq, 05)$ el experimental al control y en "negro" el \\
control al experimental.
\end{tabular}

\section{Discusión}

El objetivo fue el estudio del cambio o impacto que pudiera generar una intervención educativa. Tras seis semanas de intervención con jóvenes futbolistas se puede decir que el programa mejoró en los participantes sus actitudes a favor de: "felicitar el buen juego del oponente, animar al perdedor, respetar al árbitro aún cuando se equivoca y preocuparse por el jugador lesionado incluso llegando a rechazar una clara ocasión de gol para atenderlo". La principal contrariedad del tratamiento realizado fue la falta de honestidad en el juego.

A pesar de la dificultad que entrańa tener una actitud prodeportiva en una situación adversa a la victoria (Pinhei- ro, Camerino y Sequeira, 2014) se ha demostrado cómo es posible modificar la orientación del futbolista para tener un trato cortés con el jugador que realiza una buena jugada. Así lo revelan los resultados cosechados por el experimental: inicialmente en contra y tras la implementación del programa favorable.

La evolución de los jugadores del grupo experimental y control en relación con tener un "trato cortés con el perdedor" fue totalmente opuesta: los primeros al alza y los segundos en regresión. Resulta interesante destacar que en tres de las seis sesiones que completaban el programa se expuso la necesidad de ser empático bien para: ser un buen ganador (sesión 4), perdedor (sesión 5) o cortés con el oponente (sesión 6). Es- 
tos resultados confirman la estrecha relación entre empatía y prodeportividad, hecho que ya habían comprobado Sezen y Yildiran (2012).

Los participantes en el programa mejoraron el respeto al árbitro más que los que no recibieron tratamiento, este hallazgo resulta ser toda una hazaña en el terreno futbolístico al tratarse de una disciplina en la que el fenómeno de la violencia hacia el colegiado es una realidad latente (Kavussanu et al., 2009). Los resultados en este caso coinciden con los trabajos de Brackenridge, Pitchford y Wilson (2011) y Gimeno, Sáenz, Ariño y Aznar (2007).

Otro de los aspectos de la deportividad que más se beneficiaron del programa fue la atención y preocupación por el jugador lesionado, incluso llegando a rechazar una clara ocasión de gol. Los resultados son francamente valiosos ya que se oponen a otros trabajos que expusieron cómo la conducta antideportiva era un fenómeno omnipresente en el fútbol (Kavussanu et al., 2009; Shields, LaVoi, Bredemeier y Power, 2007).

Partiendo de los resultados obtenidos en la prueba de Willcoxon en el estudio de rangos positivos y negativos, se puede ampliar la mejora a otros matices de la deportividad que abalan aún más si cabe la eficacia del programa. Se aprecia mejorías que complementan a las anteriormente mencionadas en: "la cortesía con el vencedor y el entrenador oponente" y "consideración con el jugador que llega tarde al partido".

Los resultados obtenidos se identifican con los programas que lograron mejorar las conductas prodeportivas (Gutiérrez, 2008) o comportamientos asertivos (Cecchini et al., 2009) en el fútbol base. Los logros contrastados con otros programas reafirman el valor de estos en la promoción de la deportividad en el fútbol.

Se observó una tendencia negativa en los valores del grupo control en cinco de los once ítems de la escala. Estos resultados pueden interpretarse como un indicativo de que la evolución natural del joven futbolista es a ir empeorando su deportividad a medida que avanza la temporada y ponen de manifiesto lo importante que es el trabajo de educación en valores en escuelas deportivas, sin el cual difícilmente podremos educar a través del deporte. Papageorgiou, Hassandra y Hatzigeorgiadis (2008) ya advertían de que la competición generaba en el deportista preferencias por la victoria por encima de jugar bien o jugar limpio y que esta orientación, favorecía la proliferación de conductas antideportivas.

El programa no logró mejorar aspectos como: "obedecer al árbitro", "prestar material deportivo" y "rectificar una situación injusta para el ponente”. Es más, para el último ítem mencionado, los valores empeoraron en ambos grupos. Fue de hecho el que obtuvo la puntuación más baja del estudio.

Si interpretamos la falta de obediencia como una forma de engaño y no de respeto y la incapacidad de rectificar una situación injusta como ausencia de franqueza en el juego, en- tonces el principal revés del programa fue la ineficacia en la mejora de la honestidad del futbolista. A la luz de los resultados obtenidos se puede decir que la sinceridad en el deporte se presenta como un valor más reticente al cambio y por tanto más arraigado en el deportista que el respeto, caballerosidad o ayuda. Los resultados en este caso coinciden con investigaciones que argumentan la ardua misión de modificar actitudes de engaño en el deporte por la tendencia automática del ser humano de servir al propio interés (Shalvi, Eldar y Berby-Meyer, 2012).

Sería interesante realizar un estudio prolongado en el tiempo que recogiese al menos las categorías en las que el jugador se encuentra en un período crítico de desarrollo: benjamín, alevín e infantil. Realizar un estudio longitudinal y analizar si es posible que la tendencia hacia la victoria que va adquiriendo el jugador con la edad no se oponga al juego limpio (Papageorgiou et al., 2008) sino que lo incluya y fomente. En relación con los destinatarios, abarcar al mayor número de agentes sociales que rodean al futbolista: entrenadores, aficionados, familiares y árbitros. Y cumplimentar la valoración de actitudes con instrumentos observacionales, ya que como expusieron Lee y Cook (1990), en la medida de las actitudes existe un margen de error generado por la tendencia de los sujetos a dar respuestas socialmente favorables.

En síntesis, el programa mejoró en los participantes (grupo experimental) los componentes sociales de la deportividad. Por su parte, en el grupo con el que no se intervino (control) se observó una pérdida de sus percepciones de conductas de fair play.

\section{Aplicaciones prácticas}

Se ha demostrado como en un tiempo relativamente corto es posible mejorar la deportividad del futbolista en el trato con el oponente y respeto al colegiado. Estos resultados son esperanzadores de cara a la implantación real del juego limpio en el fútbol base y dan pautas para su pronta incorporación en planificaciones deportivas. De no ser así, también se ha expuesto cómo la evolución natural del jugador es a ir deteriorando la moral, dańando claramente la imagen del fútbol de cantera.

Este trabajo invita a técnicos y entrenadores de fútbol base a incluir en sus planificaciones deportivas contenidos que promuevan el desarrollo moral. Se especifican las metas que han de guiar el proceso, competencias básicas, evaluación y temporalización (anexo 1). Tras haberse analizado previamente a través de la observación de conductas de fair play y contrastado bibliografía de ética de las virtudes se establecieron como principales bloques de trabajo: el respeto al colegiado, la cortesía y ayuda al oponente. En el estudio realizado se logró mejorar el respeto al colegiado, cortesía y ayuda al jugador lesionado. La principal dificultad que se encontró fue 
la mejora de la deportividad cuando las acciones perjudican los intereses personales o cuando éstas aun siendo injustas suponen un beneficio para el deportista.

Resulta interesante mencionar de cara a futuros programas o campañas de promoción de fair play los principios en los que se basó este trabajo: 1. Agentes de dirigir el programa: compromiso y coordinación. 2. Aspectos actitudinales: buen clima de trabajo y habilidades sociales. 3 . Principios didácticos: recursos didácticos de sencilla lectura y fácil aplicación, testimonios de deportistas, participación activa, tiempos de reflexión, orientación en la adquisición de compromisos y su seguimiento. 4. Planificación: contextualización, establecimiento de tiempos específicos para su tratamiento, organización temporal de los contenidos, adecuación temporal del programa a los objetivos y madurez de los participantes; y valoración semanal y final de los logros.

Agradecimientos: Agradecer la colaboración de la Universidad de Extremadura, la E.U. de Magisterio "La Inmaculada", la Federación Gaditana de Fútbol, el Ciclo de Grado Superior de Animación y Actividad Física del IES Seritium de Jerez de la Frontera y la generosa acogida y participación de directivos, entrenadores, familiares y jugadores de los clubes y asociaciones deportivas: Distrito La Granja, Jerez CF Alternativa, Jerez Industrial CF, La Jerezana UD, Los Naranjos Atlético, Marianistas AD CD, ED Pago San José, Pueblo Nuevo la Granja CD AD, San Benito CF, San José Obrero UD, Veteranos Xerez CD y Xerez CD.

\section{Bibliografía}

1. Aristóteles (1972). Moral, a Nicómaco. Madrid: Espasa Calpe (6a Ed.).

2. Ato, M., y Vallejo, G. (2007). Diseños experimentales en psicología. Madrid: Pirámide.

3. Bandura, A. (1986). Social foundations of thought and action: a social cognitive theory. Englewood: Prentice Hall.

4. Belando, N., Ferriz-Morell, R., y Moreno-Murcia, J.A. (2012). Propuesta de un modelo para la mejora personal y social a través de la promoción de la responsabilidad en la actividad físico-deportiva. International Journal of Sport Science, 29, 202-222.

5. Brackenridge, C., Pitchford, A., y Wilson, M. (2011). Respect: results of a pilot project designed to improve behavior in English football. Managing Leisure, 16, 175-191. Doi: 10.1080/13606719.2011.583406.

6. Cecchini, J.A., González, C., Alonso, C., Barreal, J.M., Fernández, C., García, M.,...Nuño, P. (2009). Repercusiones del Programa Delfos sobre los niveles de agresividad en el deporte y otros contextos de la vida diaria. Apunts, 96, 34-41.

7. Cook, T.D., y Campbell, D.T. (1979). Quasi-experimentation. Desing E analysis issues for field settings, Boston: Houghton Miffin Company.

8. Comité Internacional de Fair play (2012). The essence of fair play and history. Recuperado de: http: //www.fairplayinternational.org.

9. Comité Olímpico Internacional (2001). Manual de Administración Deportiva. Solidaridad Olimpica. Lausana: Comité Olímpico Internacional.

10. Danish, S.J. (1997). Going for the goal: A life skills program for adolescent. En G. Albee y T. Gullota (Eds.) Primary preventions work, Vol. 6: Issues in children's and families' live Thousand Oaks, CA: Sage, 291-312.

11. Gimeno, F., Sáenz, A., Ariño, J.V., y Aznar, M. (2007). Deportividad y violencia en el fútbol base: un programa de Descripción y evaluación preliminar de un programa de evaluación y de prevención de partidos de riesgo. Revista de Psicología del Deporte, 16(1), 103-118.

12. Gutiérrez, D. (2008). El instrumento de observación de fair play en fútbol como herramienta para evaluar las conductas relacionadas con el juego limpio en jugadores jóvenes de fútbol. EduPsykhé. Revista de psicología y educación, 7(1), 61-79.

13. Harvey, S., Kirk, D., y O’Donovan, T.M. (2014). Sport Education as a pedagogical application for ethical development in physical education and youth sport. Sport, Education and Society, 19(1), 41-62. Doi: 10.1080/13573322.2011.624594.

14. Hechenberger, A. (2004). Sports4Peace - Ein Powerplay für den Frieden. Bewegungserziehung, 58(5), 23-28.
15. Hellison, D.R. (1995). Teaching personal and social responsibility through physical activity. Champaign, Il: Human Kinetics.

16. Kavussanu, M., Stamp, R., Slade, G., y Ring, C. (2009). Observed Prosocial and Antisocial Behaviors in Male and Female Soccer Players. Journal of Applied Sport Psychology, 21(1), 62-76.

17. Kohlberg, L. (1969). Stage and sequence: The cognitive-developmental approach to socialization. En Goslin, D.A. (Ed.). Handbook of socialization theory and research (pp. 347-480). Chicago: Rand McNally.

18. Lamoneda, J. (2014). Factores determinantes de la deportividad del joven futbolista. VIII Congreso Internacional de la Asociación Española de Ciencias del Deporte. Cáceres, España, 13-15 de Noviembre.

19. Lamoneda, J., García, A.V. Córdoba, L.G., y Vizuete, M. (2014). Detección y valoración de conductas de fair play en el fútbol alevín gaditano. E-balonmano.com: Revista de Ciencias del Deporte, 10(1), 41-52.

20. Lamoneda, J., Huertas, F.J., Córdoba, L.G., y García, A.V. (2014). Adaptación de la Escala Multidimensional de Orientaciones hacia la Deportividad al contexto del fútbol alevín. Cuadernos de Psicología del Deporte, 14(2), 71-80.

21. Lee, M.J., y Cook, C. (1990). Review of the literature on fairplay with special reference to children's sport. Comunicación presentada en el Council of Europe ameeting on Fairplay in Children's Sport, París.

22. Lemyre, P.N., Roberts, G.C., y Ommundsen, Y. (2002). Achievement goal orientations, perceived ability and Sportspersonship in youth soccer. Journal of applied sport psychology, 14, 120-136.

23. Meier, M., y Koenen, K. (2010). The Influence of Sporting Role Models on the Moral Development and Prosocial Behaviour of Children and Youth. ICSSPE Bulletin, 60, 11.

24. Papageorgiou, M., Hassandra, M., y Hatzigeorgiadis, A.I. (2008). FairPlay in Physical Education: Effects of Sex, Age and Intrinsic Motivation. Inquiries in Sport \& Physical Education, 6(3), 162-167.

25. Pinheiro, V., Camerino, O., y Sequeira, P. (2014). El fair play en la iniciación deportiva, un estudio con entrenadores de fútbol. Retos. Nuevas tendencias en Educación Física, Deporte y Recreación, 25, 32-35.

26. Ramos, J.L. (2011). Investigación cuasiexperimental. En S. Cubo, B. Martín \& J.L. Ramos (Coord.) Métodos de investigación y análisis de datos en ciencias sociales y de la salud (pp. 329-371). Madrid: Pirámide.

27. Ruiz, L.M., Rodríguez, P., Martinek, T., Schilling, T., Durán, L.J., y Jiménez, P. (2006). El Proyecto Esfuerzo: un modelo para el desarrollo de la responsabilidad personal y social a través del deporte. Revista de Educación, 341, 933-958.

28. Sáenz, A., Gimeno, F., Gutiérrez, H., y Garay, B. (2012). Prevención 
de la agresividad y la violencia en el deporte en edad escolar: Un estudio de revisión. Cuadernos de Psicología del Deporte, 12(2), 57-72.

29. Sezen-Balcikanli, G., y Yildiran, I. (2012). Sportspersonship Orientation and Empathy: a Study of Professional Football Players. Journal of Physical Education \& Sport, 12(1), 18-24.

30. Shalvi, S., Eldar, O., y Bereby-Meyer, Y. (2012). Honesty requires time (and lack of justifications). Psychological Science, 23, 1264-1270.
31. Shields, D.L., LaVoi, N.M.., Bredemeier, B.L., y Power, F.C. (2007). Predictors of Poor Sportspersonship in Youth Sports: Personal Attitudes and Social Influences. Journal of Sport \& Exercise Psychology, 29(6), 747.

32. Vallerand, R.J., Brière, N.M., Blanchard, C., y Provencher, P. (1997). Development and validation of the multidimensional Sportspersonship orientations scale. Journal of Sport and Exercise Psychology, 19, $197-$ 206.

\title{
Anexo 1. \\ Síntesis del Programa para la mejora de la componente social de la deportividad
}

\author{
1. Respetar a otros jugadores. \\ 2. Dar la mano al jugador al que le has hecho falta. \\ 3. Tras la victoria: mostrar respeto hacia el otro equipo y reconocer el buen juego del contrario. \\ 4. Al perder: reconocer el buen juego del oponente y felicitar las buenas acciones del otro equipo. \\ 5. Gane o pierda: dar la mano siempre al finalizar el partido. \\ 6. Desear un buen partido al otro equipo dando la mano al empezar (cordialidad). \\ О) 7. Respetar al árbitro. Dirigirse a él con cortesía: no discutir, insultar, o protestar. \\ 8. Colaborar con el árbitro. Reconocer las faltas levantando la mano. \\ 9. Atender al jugador lesionado: tirar el balón fuera, interesarse por él (ayuda). \\ 10. Ser generoso con los demás (prestar material deportivo). \\ 11. Reaccionar ante actuaciones injustas (actitud crítica).
}

1. La deportividad con otros jugadores al empezar y finalizar el encuentro: en la victoria y derrota.

2. La deportividad con otros jugadores durante el partido: valorar al oponente.

2.1. El valor del juego limpio en el fútbol.

2.2. Respeto al resto de jugadores.

2.3. Estrategias para la resolución pacífica de conflictos: dar la mano tras cometer una falta.

of 2.4. Aceptar, reconocer y felicitar el buen juego del otro equipo.

3. El respeto al colegiado: seguir sus indicaciones sin protestar, realizar un mal gesto o insultar, incluso si se equivoca.

3.1. El valor del árbitro.

3 3.2. La violencia en el fútbol: agresión al árbitro.

3.3. Estrategias para la mejora de la comunicación con el árbitro: diálogo asertivo.

4. Consideración por el jugador que llega tarde al encuentro.

5. Atención, consideración y ayuda por el jugador lesionado.

6. Actitud crítica: reaccionar deportivamente ante actuaciones injustas.

7. Generosidad: prestar equipamiento al oponente

1. Lingüística: participación activa en debates y comentarios críticos, resolución de problemas a través del diálogo, intercambios comunicativos respetuosos durante las sesiones, desarrollo de habilidades de comunicación asertiva en el trato con el colegiado.

2. Social y ciudadana: desarrollo de habilidades sociales (respeto, honestidad, sociabilidad, ayuda).

3. Cultural y artística: fomento de una cultura deportiva crítica ante el deporte de élite como fenómeno de masas.

4. Aprender a aprender: aprendizaje de estrategias para la mejora de las relaciones sociales y habilidades para la resolución de conflictos de forma pacífica. 
Expresa el grado de acuerdo o desacuerdo con cada una de las siguientes cuestiones con una puntuación desde 1 a 5, siendo: (1) No se corresponde conmigo en absoluto, (2)Prácticamente no se corresponde conmigo, (3) Se corresponde conmigo en parte, (4) Se corresponde conmigo en gran medida (5) Se corresponde exactamente conmigo.

1. Cuando pierdes claramente un partido, ¿̇le das la mano al vencedor admitiendo la derrota?

2. Al terminar el partido, ¿̇le das la mano al entrenador del equipo contrario?

3. Si durante el juego un futbolista del otro equipo hace una jugada fantástica, ¿̨le felicitas?

4. Cuando ganas un partido, ¿animas a los jugadores del otro equipo que ha perdido?, no se trata solo de darle la

mano, sino por ejemplo decirle que han jugado muy bien a pesar de perder.

5. Cuando el árbitro pide que te coloques más atrás en una barrera, que respetes la línea de banda en un saque o

que abandones el campo, ¿le obedeces sin protestar?

6. Cuando el árbitro se equivoca claramente en tu contra, ¿le respetas?

7. ¿Permitirías jugar a un futbolista del otro equipo que ha llegado tarde?

8. Cuando un jugador del otro equipo se lesiona, ¿te acercas a él para preocuparte por su situación?, ¿tratas de ayudarlo?

9. Imagina que durante un partido le das al balón con la mano y el árbitro no se da cuenta, ¿qué harías?: ¿̧hablarías con él para que rectifique ya que es injusto para el otro equipo?

10. Si estas en el banquillo sin jugar, ¿serías capaz de dejarle tus espinilleras o botas a un futbolista del otro equipo que se le han olvidado para que pueda jugar?

11. ¿Echarías el balón fuera para que atendiesen a un jugador lesionado, renunciando a una clara ocasión de gol?

\begin{tabular}{lcccc}
\hline Sesión & Objetivos & Competencias básicas & Contenidos & Evaluación \\
\cline { 2 - 6 } & 1,2 & $1,2,3,4$ & 2 & 1 \\
\hline 1. Juega limpio: respeta & 7,8 & $1,2,3,4$ & 3 & 5,6 \\
\hline 2. Juega limpio: respeta al árbitro & 9,11 & $1,2,3$ & 6 & 9 \\
\hline 3. Juega limpio: se honesto & $3,5,6$ & $1,2,3,4$ & 1,2 & 3,4 \\
\hline 4. Ser un buen ganador & $4,5,6$ & $1,2,3,4$ & 1,2 & 1,3 \\
\hline 5. Ser un buen perdedor & $9,10,11$ & $1,2,3,4$ & $4,5,6,7$ & $2,7,8,10,11$ \\
\hline 6. Cordialidad & & & & \\
\hline
\end{tabular}


